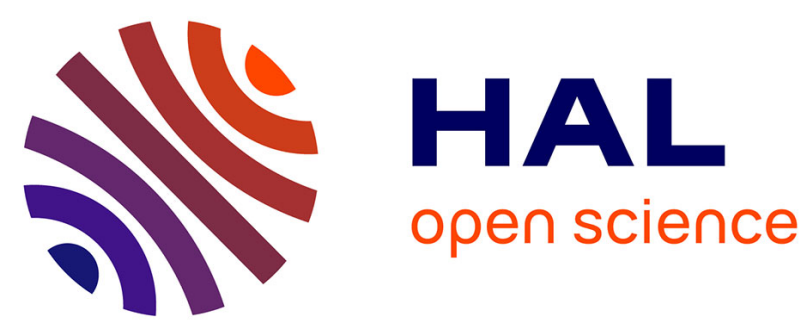

\title{
A millisecond pulsar discovery in a survey of unidentified Fermi $\gamma$-ray sources with LOFAR
}

Z. Pleunis, C.G. Bassa, J.W.T. Hessels, V.I. Kondratiev, F. Camilo, I. Cognard, J. -M. Griessmeier, B.W. Stappers, A.S. van Amesfoort, S. Sanidas

\section{- To cite this version:}

Z. Pleunis, C.G. Bassa, J.W.T. Hessels, V.I. Kondratiev, F. Camilo, et al.. A millisecond pulsar discovery in a survey of unidentified Fermi $\gamma$-ray sources with LOFAR. Astrophys.J.Lett., 2017, 846 (2), pp.L19. 10.3847/2041-8213/aa83ff . hal-01645729

\section{HAL Id: hal-01645729 \\ https://hal.science/hal-01645729}

Submitted on 10 Dec 2019

HAL is a multi-disciplinary open access archive for the deposit and dissemination of scientific research documents, whether they are published or not. The documents may come from teaching and research institutions in France or abroad, or from public or private research centers.
L'archive ouverte pluridisciplinaire HAL, est destinée au dépôt et à la diffusion de documents scientifiques de niveau recherche, publiés ou non, émanant des établissements d'enseignement et de recherche français ou étrangers, des laboratoires publics ou privés. 


\title{
A Millisecond Pulsar Discovery in a Survey of Unidentified Fermi $\gamma$-Ray Sources with LOFAR
}

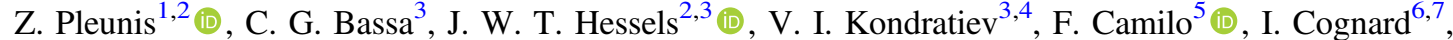 \\ J.-M. Grießmeier ${ }^{6,7}$, B. W. Stappers ${ }^{8}$, A. S. van Amesfoort ${ }^{3}$, and S. Sanidas ${ }^{2}$ \\ ${ }^{1}$ Department of Physics and McGill Space Institute, McGill University, 3600 rue University, Montréal, QC H3A 2T8, Canada; ziggy.pleunis@physics.mcgill.ca \\ 2 Anton Pannekoek Institute for Astronomy, University of Amsterdam, Science Park 904, 1098 XH Amsterdam, The Netherlands \\ ${ }^{3}$ ASTRON, the Netherlands Institute for Radio Astronomy, Postbus 2, 7990 AA Dwingeloo, The Netherlands \\ ${ }^{4}$ Astro Space Centre, Lebedev Physical Institute, Russian Academy of Sciences, Profsoyuznaya Str. 84/32, Moscow 117997, Russia \\ ${ }^{5}$ SKA South Africa, Pinelands 7405, South Africa \\ ${ }^{6}$ Laboratoire de Physique et Chimie de l'Environnement et de l'Espace, Université d'Orléans/CNRS, F-45071 Orléans Cedex 02, France \\ ${ }^{7}$ Station de radioastronomie de Nançay, Observatoire de Paris, CNRS/INSU, F-18330 Nançay, France \\ 8 Jodrell Bank Centre for Astrophysics, School of Physics and Astronomy, University of Manchester, Manchester M13 9PL, UK \\ Received 2017 June 5; revised 2017 July 25; accepted 2017 August 2; published 2017 September 5
}

\begin{abstract}
Using LOFAR, we have performed a very-low-frequency $(115-155 \mathrm{MHz})$ radio survey for millisecond pulsars (MSPs). The survey targeted 52 unidentified Fermi $\gamma$-ray sources. Employing a combination of coherent and incoherent dedispersion, we have mitigated the dispersive effects of the interstellar medium while maintaining sensitivity to fast-spinning pulsars. Toward 3FGL J1553.1+5437 we have found PSR J1552+5437, the first MSP to be discovered (through its pulsations) at a radio frequency $<200 \mathrm{MHz}$. PSR J1552 +5437 is an isolated MSP with a $2.43 \mathrm{~ms}$ spin period and a dispersion measure of $22.9 \mathrm{pc} \mathrm{cm}^{-3}$. The pulsar has a very steep radio spectral index $(\alpha<-2.8 \pm 0.4)$. We obtain a phase-connected timing solution combining the 0.74 years of radio observations with $\gamma$-ray photon arrival times covering 7.5 years of Fermi observations. We find that the radio and $\gamma$-ray pulse profiles of PSR J1552+5437 appear to be nearly aligned. The very steep spectrum of PSR J1552 +5437 , along with other recent discoveries, hints at a population of radio MSPs that have been missed in surveys using higher observing frequencies. Detecting such steep spectrum sources is important for mapping the population of MSPs down to the shortest spin periods, understanding their emission in comparison to slow pulsars, and quantifying the prospects for future surveys with low-frequency radio telescopes like SKA-Low and its precursors.
\end{abstract}

Key words: gamma rays: stars - pulsars: general - pulsars: individual (PSR J1552+5437) - stars: neutron surveys

Supporting material: machine-readable table

\section{Introduction}

The Large Area Telescope (LAT; Atwood et al. 2009) on board the Fermi Gamma-ray Space Telescope has (in)directly been responsible for dozens of millisecond pulsar (MSP) discoveries ${ }^{9}$ since it began operations in 2008 (Abdo et al. 2013). Blind pulsation searches for MSPs in Fermi data are possible (Pletsch et al. 2012), but have limited sensitivity due to the low count rate of $\gamma$-ray photons, and are furthermore computationally intensive and require a priori knowledge of orbital parameters to search for MSPs in binaries. Complementary targeted radio surveys of unidentified Fermi sources have so far identified well over 50 radio-loud $\gamma$-ray MSPs by first detecting pulsed radio emission and later applying the timing model derived from radio observations to detect $\gamma$-ray pulsations (e.g., Ray et al. 2012; Camilo et al. 2015). One-third of the $3033 \gamma$-ray sources in the latest point-source catalog (3FGL) remain unidentified (most are likely blazars, though certainly some of these are undiscovered MSPs; Acero et al. 2015), indicating a clear need for continued multi-wavelength follow-up observations.

Almost all MSP surveys to date have been performed at observing frequencies of $300-2000 \mathrm{MHz}$ and higher, thereby potentially missing MSPs with very steep spectra $(\alpha<-3$, where $S \propto \nu^{\alpha}$ ) and low flux densities.

\footnotetext{
See http://tinyurl.com/fermipulsars for an overview.
}

Here, we present the results of a Low-Frequency Array (LOFAR) pilot survey at $115-155 \mathrm{MHz}$, targeted at Fermi $\gamma$-ray sources. The primary goal of the survey was to test the hypothesis that very-steep-spectrum radio MSPs have been missed in previous pulsar surveys (both targeted and all-sky). This is important, e.g., for determining whether MSPs and slow pulsars have similar spectral index distributions-a key observable related to the underlying emission mechanism (e.g., Bates et al. 2013). Such searches are also motivated by the hypothesis that the fastest-spinning MSPs are also preferentially steeper spectrum. Mapping the MSP spin distribution is important for understanding the pulsar recycling mechanism, and probing beyond the currently known highest spin rate of $716 \mathrm{~Hz}$ (Hessels et al. 2006) could reach the regime where rotation-derived neutron star radius limits become constraining. We outline our survey strategy, observations and analysis in Section 2. In Section 3 we present the results. We discuss the results and conclude in Section 4.

\section{LOFAR Survey of Unidentified $\gamma$-Ray Sources}

\subsection{Survey Setup}

We have used the LOFAR High Band Antennas (HBAs; van Haarlem et al. 2013) of 21 of the 24 LOFAR Core stations ${ }^{10}$ to

\footnotetext{
${ }^{10}$ We excluded station CSO13 because it had a $45^{\circ}$ dipole rotation error at the time of the observations and the two outermost stations CS103 and CS302 to be able to cover the error ellipses of a larger number of Fermi sources.
} 


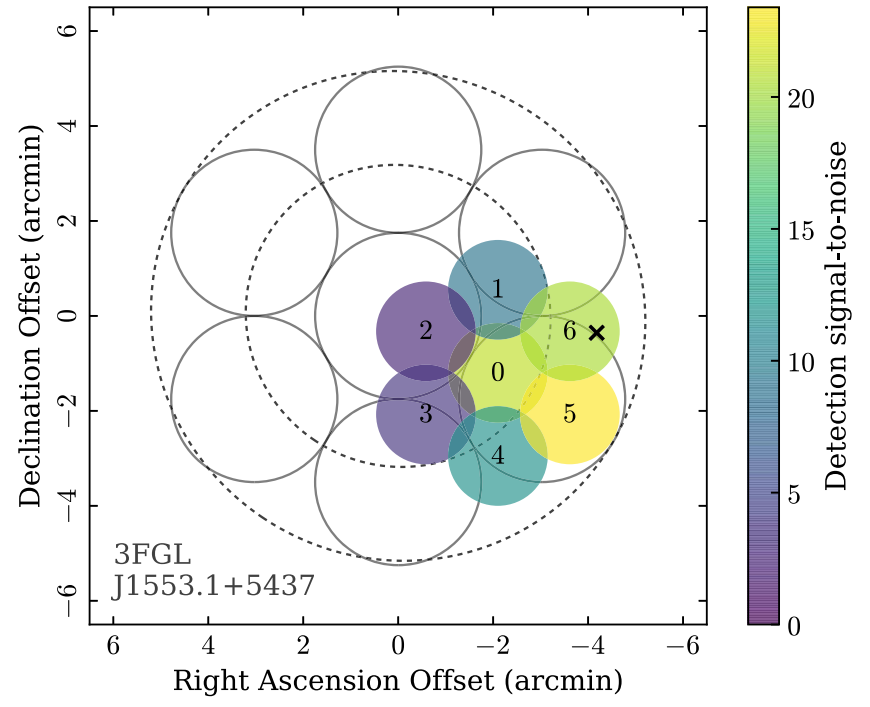

Figure 1. Schematic representation of LOFAR tied-array beam positions for the observations of 3FGL sources. Gray open circles indicate the FWHM of the beams in the search observations. Real beams have side lobes and are elongated for non-zero zenith angles. The $68 \%$ and $95 \%$ confidence error ellipses from the third Fermi point-source catalog are depicted with gray dashes. The filled circles represent the beams from the confirmation observation of PSR J1552 +5437 , with the color indicating the signal-to-noise of the folded pulsar signal. The confirmation observation used all LOFAR Core stations, and thus has a higher sensitivity in the center of the beams than the discovery observation. The pulsar's best-fit position from radio timing is denoted with a black cross.

form 7 tied-array beams (Stappers et al. 2011). This observational setup has baselines up to $2.3 \mathrm{~km}$ and provides tied-array beams of $\sim 3 ! 5$ in diameter (FWHM) at the central frequency, with 7 beams covering a total circular field-of-view (FoV) of about $10^{\prime}$ in diameter (see Figure 1). With this setup we have observed 52 out of 1010 unidentified $\gamma$-ray sources from the 3FGL Fermi-LAT point-source catalog (Acero et al. 2015). These 52 sources were selected as they are visible to LOFAR (source elevation $>30^{\circ}$ during transit), located away from the Galactic plane $\left(|b|>10^{\circ}\right.$; where the sky temperature and scattering at $135 \mathrm{MHz}$ are significantly lower), and because they have positional uncertainty regions less than $10^{\prime}$ in diameter (i.e., fit the FoV of 7 tied-array beams). No cuts on the spectral parameters of the sources were performed. The observed sources and some of their parameters are listed in Table 1. The sample of Fermi sources searched here does not overlap with that of Bassa et al. (2017b).

We employed a semi-coherent dedispersion scheme, aimed at mitigating the effects of dispersive smearing and implemented in comt (Bassa et al. 2017a). To allow coherent dedispersion, we have recorded complex voltage data for dual-polarization, Nyquist sampled subbands of $195.3125 \mathrm{kHz}$ bandwidth (5.12 $\mu \mathrm{s}$ sampling). To maximize sensitivity and FoV we have used signals from 200 subbands in the 115 $-155 \mathrm{MHz}$ frequency range (39.06 $\mathrm{MHz}$ bandwidth). Modest integration times of $T_{\mathrm{obs}}=20$ minutes were chosen to maintain sensitivity to accelerated signals from binary systems.

For each observation, the 200 frequency subbands were coherently dedispersed to 80 evenly spaced trial dispersion measures (DMs), ranging from 0.5 to $79.5 \mathrm{pc} \mathrm{cm}^{-3}$ (about twice the expected maximum Galactic DM for most of the surveyed sources), and channelized into a total of 1600 channels, using cdmt. The time and spectral resolution after channelization were $40.96 \mu \mathrm{s}$ and $24.41 \mathrm{kHz}$, respectively.
Around each coherent DM trial we made incoherent DM trials in steps of $0.002 \mathrm{pc} \mathrm{cm}^{-3}$. The two DM step sizes are chosen to limit the total (intra-channel and $\triangle \mathrm{DM}$ ) dispersive smearing compared to the true DM of the source to a maximum of $0.15 \mathrm{~ms}$ (see the top panel in Figure 2). Each dedispersed time series was searched for accelerated periodic signals in the frequency domain, and the 200 best pulsar candidates from each beam, according to a modified version of PRESTO's accel_sift.py sifting script (Ransom 2001), were folded and inspected by eye.

Confirmation observations used all LOFAR Core stations (baselines up to $3.5 \mathrm{~km}$ ), and thus have tied-array beams with a $\sim 3$ times smaller area of $\sim 2^{\prime}$ in diameter (FWHM) at the central frequency. Furthermore, the tied-array ring size (the offset of the center of the outer beams from the center of the pointing) is reduced to $1 ! 75$, such that the beams overlap slightly, and the position of a newly discovered pulsar can be refined by weighting the signal-to-noise ratios of detections in the different beams (see the colored filled circles in Figure 1 for an illustration). However, note that the ionosphere can shift beams by approximately an arcminute during periods of strong ionospheric turbulence. This can somewhat reduce the accuracy of this positional determination method.

\subsection{Survey Sensitivity}

Although the effects of dispersive smearing within a channel can be mitigated by the use of coherent dedispersion, the sensitivity of any pulsar survey at low radio frequencies is ultimately limited by scattering (approximately $\propto \nu_{\text {obs }}^{-4}$ ), which results in an exponential broadening of the observed pulse shapes. We calculated the expected scattering times using the empirical fit for scattering as a function of DM made by Bhat et al. (2004), and compared this to dispersive smearing within channels (see the top panel in Figure 2). We have calculated the minimum detectable flux density our survey was sensitive to, using the modified radiometer equation for pulsars (Lorimer \& Kramer 2012, Appendix 1.4), where we have used $\sigma=10$ as the minimum signal-to-noise ratio for a convincing pulsar candidate (although candidates with a somewhat lower signalto-noise ratio were also investigated), $\beta \approx 1.0$ as the digitization correction factor (survey observations were processed with 8-bit integer bit depth), $T_{\text {sys }} \approx 400 \mathrm{~K}$ as the temperature of the telescope and the sky at the observing frequency, and $G \approx 5.6 \mathrm{~K} \mathrm{Jy}^{-1}$ as the telescope's gain. ${ }^{11}$ Sensitivity curves for a pulsar with a 1 and a $10 \mathrm{~ms}$ spin period and an intrinsic $10 \%$ duty cycle are shown in the bottom panel of Figure 2. Out to DMs of about $40 \mathrm{pc} \mathrm{cm}^{-3}$ we were sensitive to $2 \mathrm{~ms}$ pulsars brighter than $\sim 2 \mathrm{mJy}$, if the source was not eclipsed at the time of observation (many binary $\gamma$-ray MSPs are eclipsed for up to $\sim 50 \%$ of their orbit). This flux limit applies to observations at zenith; the sensitivity falls off approximately as $\sin ^{-1.4}\left(\theta_{\mathrm{z}}\right)$, where $\theta_{\mathrm{z}}$ is the zenith angle (Noutsos et al. 2015).

\section{Discovery and Timing of PSR J1552+5437}

We discovered an isolated pulsar with a $2.43 \mathrm{~ms}$ spin period at a DM of $22.9 \mathrm{pc} \mathrm{cm}^{-3}$, in a pointing toward 3FGL J1553.1 +5437 (see Figure 3 for its radio and $\gamma$-ray pulse profile). The

\footnotetext{
11 The gain $G \sim T_{\text {sys }} / \mathrm{SEFD}_{\text {Core }} \approx 400 \mathrm{~K} /(3000 \mathrm{Jy} / 42) \approx 5.6 \mathrm{~K} \mathrm{Jy}^{-1}$ when using 21 of the LOFAR Core stations (van Haarlem et al. 2013); here, SEFD is the system equivalent flux density.
} 
Table 1

LOFAR Survey of Unidentified Fermi-LAT Sources: Source Information

\begin{tabular}{|c|c|c|c|c|c|}
\hline Name & $\begin{array}{c}\text { Observation Epoch } \\
\text { (MJD) }\end{array}$ & $\begin{array}{c}\text { Altitude }^{\mathrm{a}} \\
\left({ }^{\circ}\right)\end{array}$ & $\begin{array}{l}\text { Azimuth }^{\mathrm{a}} \\
\left({ }^{\circ}\right)\end{array}$ & $\begin{array}{c}r_{95}{ }^{\mathrm{b}} \\
\left({ }^{\prime}\right)\end{array}$ & $\begin{array}{l}\text { Max. Gal. DM } \\
\left(\mathrm{pc} \mathrm{cm}^{-3}\right)\end{array}$ \\
\hline 3FGL J0017.1+1445 & 57376 & 51.9 & 184.5 & 5.08 & 37,28 \\
\hline 3 FGL J0020.9+0323 & 57376 & 40.2 & 189.5 & 3.46 & 33,23 \\
\hline 3FGL J0031.6+0938 & 57376 & 45.1 & 201.3 & 5.67 & 35,25 \\
\hline 3FGL J0032.5+3912 & 57376 & 74.9 & 209.5 & 5.3 & 61,55 \\
\hline 3FGL J0102.1+0943 & 57376 & 45.7 & 198.1 & 4.93 & 35,25 \\
\hline
\end{tabular}

Notes.

at the midpoint of the LOFAR observation.

b Semimajor axis of $95 \%$ confidence error region in the 3FGL catalog.

c According to the NE2001 (Cordes \& Lazio 2002) and the YMW16 (Yao et al. 2017) models for Galactic electron density, respectively.

(This table is available in its entirety in machine-readable form.)

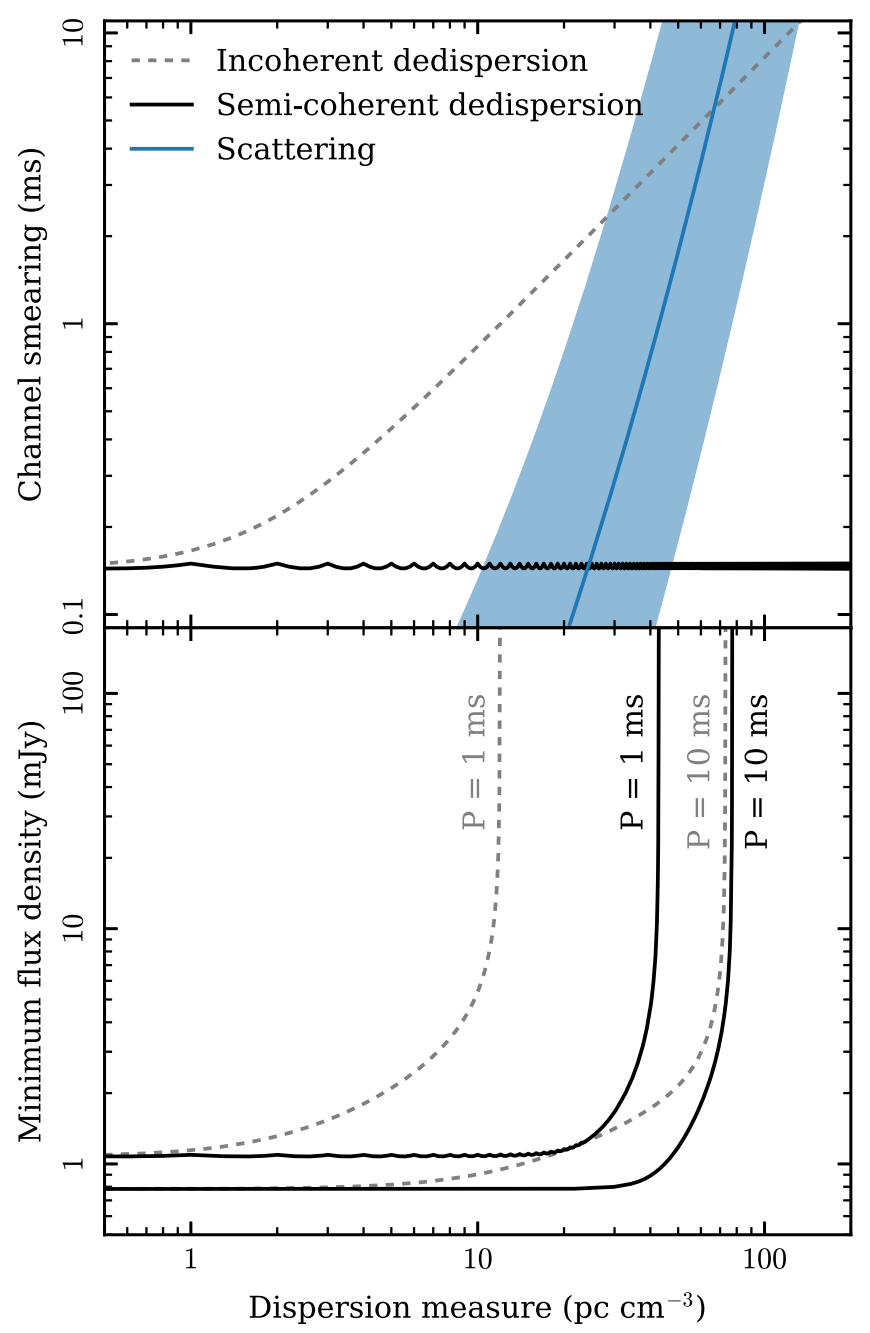

Figure 2. LOFAR targeted survey sensitivity to MSPs at a central observing frequency of $135 \mathrm{MHz}$. Top: the leftover dispersive channel smearing is depicted for incoherent (gray dashed line) and semi-coherent (black solid line; with the setup described in Section 2) dedispersion. The expected scattering time based on Bhat et al. (2004) is depicted with a blue solid line, with the blue shaded region showing up to $10 \times$ smaller and larger values, to reflect the scatter in the relation. For DMs $\gtrsim 25 \mathrm{pc} \mathrm{cm}^{-3}$ scattering becomes the dominant source of smearing within channels and scattering starts to drastically reduce the sensitivity to MSPs for DMs $\gtrsim 50 \mathrm{pc} \mathrm{cm}^{-3}$. Bottom: the minimum flux density an MSP needs to have at $135 \mathrm{MHz}$ in order to be discovered by an incoherent (gray dashed lines) and by a semi-coherent (black solid lines) search pipeline with the effect of scattering taken into account. pulsar was detected in two adjacent beams in the discovery observation.

\subsection{Radio Analysis}

Following the discovery and confirmation of the pulsar we started a timing campaign with LOFAR. Timing observations use all Core stations and the HBA bandwidth from 110 to $188 \mathrm{MHz}$, two times wider than possible in the survey observations. After initial dense and logarithmically spaced 10 minute observations spanning two weeks, the pulsar was observed once per month for 20 minutes. All observations are dedispersed and folded using the LOFAR Pulsar Pipeline (e.g., Kondratiev et al. 2016). Pulse times-of-arrival (TOAs) are extracted from 5 minute sub-integrations using tools from the PSRCHIVE $^{12}$ (Hotan et al. 2004) pulsar software package. We have used TEMPO $2^{13}$ (Hobbs et al. 2006) to obtain an initial phase-connected timing solution spanning 0.74 years and fitted for position, spin frequency, and DM (see Figure 4). The efac/equad plug-in (Wang et al. 2015) was used to rescale the LOFAR TOA uncertainties, suggesting that a multiplication factor of 1.3 and an additional uncertainty of $0.8 \mu$ s (multiplied with and added to the initial uncertainty in quadrature) better reflect the expected Gaussian scatter of the residuals. Note that scattering can influence the measured DM and that there are thus likely systematic uncertainties on the DM that are larger than the nominal TEMPO2 error listed in Table 2. Also, the frequency dependence of the pulse profile might bias the measured DM value.

The pulsar's flux density was measured in all timing observations by calibrating the observations using an improved Hamaker beam model (Hamaker 2006) and comparing the onpulse with the off-pulse window (full details of LOFAR MSP flux calibration are described by Kondratiev et al. 2016). These measurements lead to a mean flux density for 19 observations at $150 \mathrm{MHz}$ of $3.8 \pm 1.9 \mathrm{mJy}(50 \%$ uncertainty), but the observed flux density can vary by a factor $\sim 2$ from observation to observation-possibly because of refractive scintillation, though RFI and ionospheric beam jitter can also influence this. A search for the Faraday rotation measure toward the pulsar using PSRCHIVE's rmfit routine did not converge for any of the LOFAR observations, likely because the pulsar shows little or no polarization beyond the detection limit.

\footnotetext{
12 http://psrchive.sourceforge.net

13 http://sourceforge.net/projects/tempo2/
} 

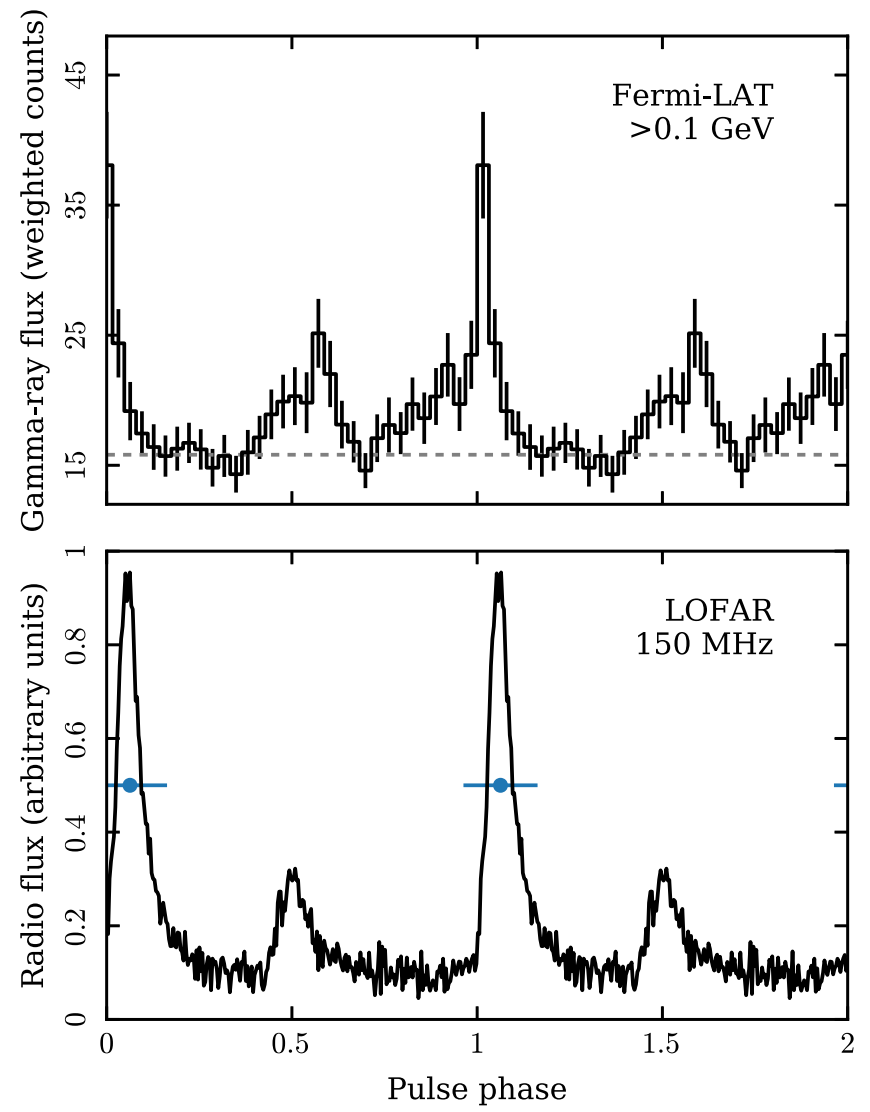

Figure 3. Aligned $\gamma$-ray and radio pulse profiles of PSR J1552+5437. The $\gamma$-profile contains $\sim 7.5$ years of Fermi photons, weighted with their probability of coming from the source, and folded in 32 phase bins. The errors on the phase bins as well as the background (gray dashed line) are estimated as in Abdo et al. (2013). The radio profile is a stacked pulse profile for 19 timing observations (total integration time of $6 \mathrm{hr}$ ) at a central frequency of $150 \mathrm{MHz}$, folded in 256 bins. The blue error bars indicate the potential radio profile phase shift due to a DM variation of $10^{-3} \mathrm{pc} \mathrm{cm}^{-3}$ over the course of the Fermi mission.

PSR J1552 +5437 was observed at L-band for $9 \times 30$ minutes and $4 \times 1 \mathrm{hr}$ with the Lovell $(400 \mathrm{MHz}$ bandwidth at $1532 \mathrm{MHz}$ center frequency) and Nançay (NRT; $512 \mathrm{MHz}$ at $1486 \mathrm{MHz}$ ) radio telescopes, but not detected, limiting the flux density to less than $13 \mu \mathrm{Jy}$, when these data sets are co-added (under the assumption that diffractive scintillation is averaged out; here, the expected scintillation bandwidth is only $\sim 10 \mathrm{MHz}$ at $1400 \mathrm{MHz}$ ). A nondetection of the pulsar at $820 \mathrm{MHz}(200 \mathrm{MHz}$ bandwidth) in a $1.5 \mathrm{hr}$ observation using the Robert C. Byrd Green Bank Telescope sets an upper limit to the pulsar's flux density at $820 \mathrm{MHz}$ of $17 \mu \mathrm{Jy}$. These upper limits are calculated using the modified radiometer equation for pulsars (Lorimer \& Kramer 2012, Appendix 1.4), assuming that PSR J1552+5437 would have been detected in those bands if it had a signal-to-noise ratio of at least 5 and a similar pulse width to our LOFAR detections. Based on the same assumptions, the upper limit for NRT is confirmed to be $20 \mu \mathrm{Jy}$ after flux calibration of the $4 \times 1 \mathrm{hr}$ of observation with a pulsed noise diode and a calibration source. The detections at $150 \mathrm{MHz}$ and the upper limits at 820 and $1400 \mathrm{MHz}$ constrain the radio power-law spectral index of PSR J1552+5437 to be $\alpha<-3.2 \pm 0.4$, where we assumed that the radiometer equation has a $50 \%$ uncertainty. A more conservative upper limit, however, takes into account the potential overestimate of LOFAR pulsar fluxes by a factor $\sim 2$ that was noted by Frail et al. (2016). In that case the LOFAR $150 \mathrm{MHz}$ flux density would be $1.9 \pm 0.9 \mathrm{mJy}$, and the upper limit on the spectral index $\alpha<-2.8 \pm 0.4$. Future observations at $350 \mathrm{MHz}$ will also be useful for mapping the spectrum.

We have also observed the pulsar with LOFAR's Low Band Antennas (LBAs) for $1 \mathrm{hr}$ at $30-90 \mathrm{MHz}$ on MJD 57496, but were unable to detect the pulsar by folding the data with the best-fit parameters derived from the timing analysis. This is unsurprising given the faintness of the source in the LOFAR HBA and the increased system temperature $T_{\text {sys }}$ in the LBA. Also, the scattering tail that is already visible in the radio profile at $150 \mathrm{MHz}$ (Figure 3) will be $\sim 10 \times$ larger in the LBA range and would smear out the pulsations. Only three (very bright, and unscattered) MSPs have so far been detected using the LOFAR LBAs (Kondratiev et al. 2016).

\section{2. $\gamma$-Ray Analysis}

We downloaded the Fermi-LAT Pass 8 photons of the SOURCE class from 2008 August 4 (the start of the mission) to 2016 October 14 , within $20^{\circ}$ of the best position derived from radio timing. Using the Fermi Science Tools, we selected the photons in the energy range $0.1-100 \mathrm{GeV}$ using the recommended cuts. We performed a binned maximum likelihood gtlike analysis on the photons in the $20^{\circ} \times 20^{\circ}$ square centered on the timing position, leaving only the spectral parameters of the sources within the inner $5^{\circ}$ radius free. Our source model was based on the 3FGL catalog and as models for the Galactic diffuse emission and isotropic diffuse background we used the gll_iem_v06.fits (Acero et al. 2016) and iso_P8R2_SOURCE_V6_V06.txt templates ${ }^{14}$, respectively. 3FGL J1553.1+5437 moved to the pulsar's timing position is detected with a test statistic TS value of 205 (about $14 \sigma$, while the source had a $\sim 8.5 \sigma$ significance previously) using an exponentially cutoff power-law model to describe its spectrum. The exponentially cutoff model is preferred over a simpler power law as $\mathrm{TS}_{\text {cut }} \equiv 2 \Delta \log$ (likelihood) $=15>9$ (likelihood ratio test, following Abdo et al. 2013), and the bestfit parameters are listed in Table 2.

Based on the spectral analysis, all the events in the region around the source were assigned a probability of originating from 3FGL J1553.1+5437 using gtsrcprob (Kerr 2011). Selecting only those events with a probability $>20 \%$ resulted in 350 photons. Pulsar rotational phases $\phi_{i}(t)$ were computed based on the radio timing solution using TEMPO2 ${ }^{15}$ (Hobbs et al. 2006) with the fermi plug-in (Ray et al. 2011). Folding the $\gamma$-ray photons over the range where the radio timing solution was valid did not result in a significant pulse profile, and we thus performed a brute-force search over the pulsar's spin frequency $f$ and spin-frequency derivative $\dot{f}$ to find a coherent solution over the 7.5 years of Fermi data (neglecting higher order effects in this search is feasible because the MSP is likely isolated).

In the brute-force search, the barycentered phases were updated using the Taylor series

$$
\phi_{i}(t)=\phi_{i, 0}+f\left(t_{i}-t_{0}\right)+\frac{1}{2} \dot{f}\left(t_{i}-t_{0}\right)^{2}
$$

\footnotetext{
14 https://fermi.gsfc.nasa.gov/ssc/data/access/lat/BackgroundModels.html

15 http://sourceforge.net/projects/tempo2/
} 

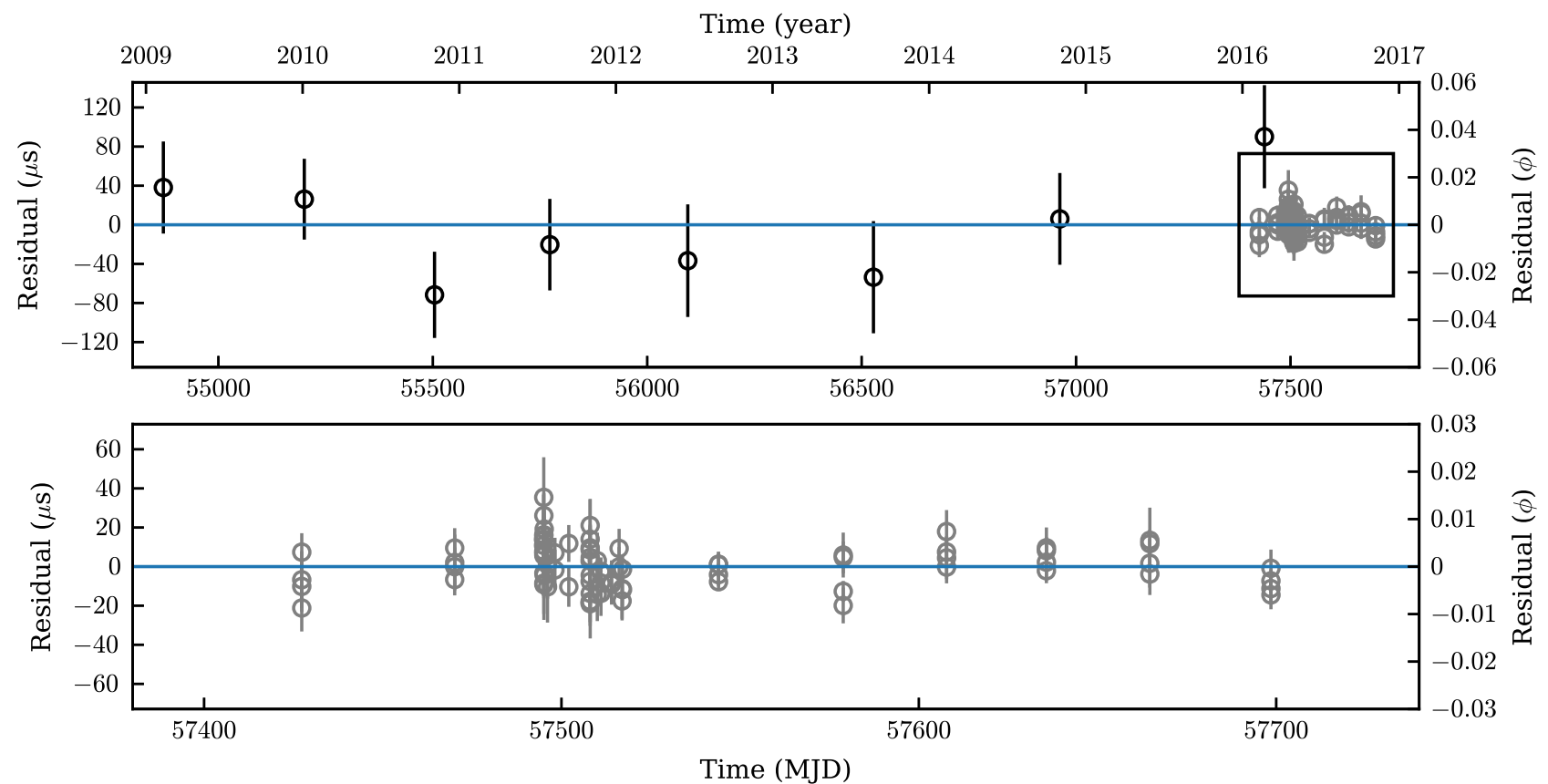

Figure 4. Timing residuals for PSR J1552+5437 as a function of time. The model is depicted with a blue line, the Fermi TOAs with black circles, and the LOFAR TOAs with gray circles. The lower panel is a magnification of the upper panel, showing only the LOFAR TOAs.

for $100 \times 100$ values of $f$ and $\dot{f}$ within two times the error range of the radio timing solution. The H-test (de Jager et al. 1989) of the folded pulse profile was calculated for each trial. With the $f$ and $\dot{f}$ that maximized $\mathrm{H}$ to 70 , it was possible to significantly fold all 350 Fermi photons, which confirms the link between PSR J1552+5437 and 3FGL J1553.1+5437.

To lift the degeneracy between astrometric and rotational parameters in the timing solution we included the $\gamma$-ray data in our timing analysis. We used an unbinned maximum likelihood method to extract 8 topocentered TOAs with at least a $3 \sigma$ detection from the 350 Fermi photons (Ray et al. 2011). More sophisticated and sensitive unbinned methods for extracting $\gamma$-ray TOAs have been developed in recent years (e.g., Kerr et al. 2015; Pletsch \& Clark 2015), but using the method described above suffices for our present purposes.

The results of joint radio and $\gamma$-ray timing are listed in Table 2, the $\gamma$-ray profile folded with the final timing solution is depicted in Figure 3, and the timing residuals as a function of time are shown in Figure 4. The timing position is not at the center of the three beams with the best detections in the confirmation observations (see Figure 1), while the timing position based on the radio data alone agrees with the full timing solution to within a few hundredths of an arcsecond. Possibly, the ionosphere has caused the beams to shift by $\sim 1^{\prime}$ in the confirmation observation. We also note that the schematic shown in Figure 1 is only a rough approximation of the true beam shapes.

The observed spin period derivative of $2.80 \times 10^{-21} \mathrm{~s} \mathrm{~s}^{-1}$ is not the intrinsic value, as it has to be corrected for the non-zero proper motion of the pulsar, the Shklovskii effect (Shklovskii 1970), and for movement due to the kinematics of the Galaxy (e.g., Nice \& Taylor 1995). The Galactic contribution is $(-4.36,-6.20) \times 10^{-22} \mathrm{~s} \mathrm{~s}^{-1}$ for a $(1.2,2.6) \mathrm{kpc}$ distance in the line-of-sight. This is the sum of the differential Galactic rotation and the $k_{\mathrm{z}}$ term. Adding this correction leads to a spinfrequency derivative of $(3.24,3.42) \times 10^{-21} \mathrm{~s} \mathrm{~s}^{-1}$. With the current data, it was not possible to significantly fit for the proper motion of the pulsar. However, the uncertainty on the fit values limits the proper motion to $<36.8{\text { mas } \mathrm{yr}^{-1}}^{-1}(3 \sigma)$, corresponding to a Shklovskii correction to the spin period derivative of $<(9.6,20.8) \times 10^{-21} \mathrm{~s} \mathrm{~s}^{-1}$. The inferred surface magnetic field strength based on the observed spin period and spin period derivative, corrected for Galactic acceleration, is with $(9.0,9.2) \times 10^{7} \mathrm{G}$ already one of the lowest pulsar magnetic fields measured to date, and will become slightly lower after correcting $\dot{P}$ with an extended timing baseline.

Finally, we consider the offsets between the radio and $\gamma$-ray pulse peaks. As can be seen in Figure 3, both the radio and the $\gamma$-ray profile show a main pulse and a subpulse offset by about half a rotational phase. The $\gamma$-ray profile does not show any additional features when the number of phase bins is increased. We have set the rotational phase $\phi=0$ at the onset of the main pulse of the LOFAR radio profile. To quantify the peak separations, we fitted a Gaussian profile to both radio pulses. The peak around phase 0 in the probability-weighted $\gamma$-ray profile was fitted using two Lorentzian profiles, and the other pulse with one Lorentzian profile, on top of the background. The maximum of the radio profile is at $\phi_{\mathrm{r}}=0.063 \pm 0.002$ (where the rotational phase is defined between 0.0 and 1.0, and errors are statistical), the radio subpulse peaks at $\phi=0.51 \pm 0.01$, and the peaks of the $\gamma$-ray profiles are at $\phi_{1}=0.021 \pm 0.004$ and $\phi_{2}=0.553 \pm 0.013$. Adopting this $\gamma$-ray peak definition leads to a radio-to- $\gamma$-ray lag of $\delta=\phi_{1}-\phi_{\mathrm{r}} \simeq 0.04$ and a $\gamma$-ray peak separation of $\Delta=\phi_{2}-\phi_{1} \simeq 0.53$. These numbers seem consistent with other LAT MSPs (Abdo et al. 2013); the $\Delta \gtrsim 0.5$ in phase, however, might indicate that the definition of the first and second $\gamma$-ray peaks could, in principle, be reversed. If that is the case, the $\gamma$-rays either lead the radio by $\sim 0.49$ or trail it by $\sim 0.51$ in phase.

The alignment of the main peaks of the radio and $\gamma$-ray profile might be real, but it could also reflect the limited 
Table 2

Parameters for PSR J1552+5437

\begin{tabular}{ll}
\hline \hline Parameter & \multicolumn{1}{c}{ Value } \\
\hline \multicolumn{1}{c}{ Timing Parameters (Radio and $\gamma$-Ray) } \\
\hline R.A. (J2000) & $15^{\mathrm{h}} 52^{\mathrm{m}} 53.33117(17)$ \\
Decl. (J2000) & $+54^{\circ} 37^{\prime} 05 ! 7866(14)$ \\
Spin frequency $(\mathrm{Hz})$ & $411.88053142429(10)$ \\
Frequency derivative $\left(\mathrm{Hz} \mathrm{s}^{-1}\right)$ & $-4.746(17) \times 10^{-16}$ \\
Dispersion measure $\left(\mathrm{pc} \mathrm{cm}^{-3}\right)$ & $22.9000(5)$ \\
Span of timing data (MJD) & $54871.7-57698.5$ \\
Epoch of timing solution (MJD) & 56285 \\
Number of TOAs & 88 \\
rms timing residual $(\mu \mathrm{s})$ & 10.1 \\
Reduced $\chi^{2}$ value & 1.1 \\
Clock correction procedure & TT(BIPM2011) \\
Solar system ephemeris model & DE421 \\
\hline
\end{tabular}

Radio Flux Densities

\begin{tabular}{ll}
\hline Flux density at $150 \mathrm{MHz}(\mathrm{mJy})$ & $3.8 \pm 1.9$ \\
Flux density at $820 \mathrm{MHz}(\mu \mathrm{Jy})$ & $<17$ \\
Flux density at $1.4 \mathrm{GHz}(\mu \mathrm{Jy})$ & $<20$
\end{tabular}

\begin{tabular}{|c|c|}
\hline \multicolumn{2}{|c|}{ Derived Parameters } \\
\hline Spin period (ms) & 2.4279 \\
\hline Spectral index & $<-2.8 \pm 0.4$ \\
\hline Galactic longitude $\left({ }^{\circ}\right)$ & 85.6 \\
\hline Galactic latitude $\left(^{\circ}\right)$ & 47.2 \\
\hline DM-derived distance $^{\mathrm{a}}(\mathrm{kpc})$ & $1.2,2.6$ \\
\hline Spin-down luminosity ${ }^{\mathrm{a}, \mathrm{b}}\left(\mathrm{erg} \mathrm{s}^{-1}\right)$ & $(8.9,9.4) \times 10^{33}$ \\
\hline Surface magnetic field ${ }^{\mathrm{a}, \mathrm{b}}(\mathrm{G})$ & $(9.0,9.2) \times 10^{7}$ \\
\hline Characteristic age agears) $^{\mathrm{a}, \mathrm{b}}$ (year & $(1.2,1.1) \times 10^{10}$ \\
\hline \multicolumn{2}{|c|}{$\gamma$-Ray Parameters } \\
\hline$\gamma$-ray-radio profile lag $(\phi)$ & $0.042 \pm 0.004 \pm 0.1$ \\
\hline$\gamma$-ray peak separation $(\phi)$ & $0.53 \pm 0.01$ \\
\hline$\gamma$-ray photon index & $1.4 \pm 0.3$ \\
\hline$\gamma$-ray cutoff energy $(\mathrm{GeV})$ & $3.7 \pm 1.6$ \\
\hline Photon flux $\left(\mathrm{cm}^{-2} \mathrm{~s}^{-1}\right)$ & $(2.5 \pm 0.9) \times 10^{-9}$ \\
\hline Energy flux ( $\left.\mathrm{erg} \mathrm{cm}^{-2} \mathrm{~s}^{-1}\right)$ & $(2.7 \pm 0.4) \times 10^{-12}$ \\
\hline Luminosity $^{\mathrm{a}}\left(10^{32} \mathrm{erg} \mathrm{s}^{-1}\right)$ & $(4.7 \pm 0.7),(22 \pm 3.2)$ \\
\hline Efficiency $^{\mathrm{a}}(\%)$ & $6.1,28.6$ \\
\hline
\end{tabular}

Notes.

a Based on the NE2001 (Cordes \& Lazio 2002) and the YMW16 (Yao et al. 2017) models, respectively.

${ }^{b}$ Upper limit: corrected for the acceleration due to the kinematics of the Galaxy, but not for the Shklovskii effect.

baseline of the radio timing of the pulsar. A $10^{-3} \mathrm{pc} \mathrm{cm}^{-3}$ variation in DM over the length of the Fermi mission could lead to a shift of $\sim 0.1$ in rotational phase between the radio and the $\gamma$-ray profiles. Such a DM variation would be consistent with those seen for other MSPs (Keith et al. 2013). A higher-frequency radio profile (e.g., measured at $820 \mathrm{MHz}$ ) would be less sensitive to DM variations, but we have so far been unable to detect PSR J1552+5437 at higher radio frequencies.

\section{Discussion and Outlook}

In our targeted LOFAR survey toward 52 unidentified Fermi-LAT $\gamma$-ray sources, we discovered one MSP. The newly discovered MSP, PSR J1552+5437, has a low inferred magnetic field $\left(B<9.2 \times 10^{7} \mathrm{G}\right)$ and a very steep power-law radio spectrum $(\alpha<-2.8 \pm 0.4)$. Only 9 pulsars in the ATNF pulsar catalog ${ }^{16}$ have lower inferred magnetic fields (Manchester et al. 2005), and only 8 of the 200 GMRTdetected pulsars have spectral indices $<-2.8$ (Frail et al. 2016). In $2.9 \mathrm{ks}$ of Swift-XRT observations of 3FGL J1553.1+5437 no source is detected above ${ }^{17} 3 \sigma$ (Stroh \& Falcone 2013), making the pulsar a suboptimal target for, e.g., the NICER mission (Arzoumanian et al. 2014), despite its relatively small DM-distance.

This pilot survey has shown that LOFAR is capable of discovering MSPs. In fact, it is the first digital aperture array to discover an MSP directly through its pulsed signal, and this is the lowest radio frequency $(135 \mathrm{MHz})$ at which any MSP has been discovered to date. As a follow-up survey of Fermi unidentified sources, however, it has a success rate of only a few percent, which is low compared to similar surveys at higher frequencies (which have success rates of $12 \%-26 \%$; see Cromartie et al. 2016 for an overview). A refined selection of Fermi targets (choosing the most pulsar-like unidentified $\gamma$-ray sources) will likely increase the success rate of future LOFAR MSP searches, as suggested by the recent discovery of PSR J0952-0607 (Bassa et al. 2017b). Furthermore, instead of only once, each source should be observed two or three times to reduce the probability of catching the pulsar during an eclipse.

Nonetheless, 3FGL J1553.1+5437 is a relatively weak Fermi point source with a relatively large positional uncertainty that was classified as a likely active galactic nucleus using machine-learning techniques (Saz Parkinson et al. 2016). Targeted radio surveys often favor the bright and wellconstrained $\gamma$-ray sources with weaker MSPs in unidentified Fermi sources going unnoticed. This was also observed in a recent blind search for $\gamma$-ray pulsars in Fermi data, where at least two pulsars were discovered close to or slightly outside the edge of the search region (Clark et al. 2017). This, and the fact that PSR J1552+5437 was not detected at $820 \mathrm{MHz}$ and $1.4 \mathrm{GHz}$, advocates repeat searches of Fermi unidentified sources-even those that a priori appear less pulsar-like-using low-frequency radio telescopes and covering a reasonable region around the quoted positional uncertainty.

PSR J1552+5437 shows characteristics also seen in other MSPs with nearly aligned radio and $\gamma$-ray profiles. For this class of MSPs, models have been developed where both the radio and $\gamma$-ray emission are produced close to the light cylinder, with the radio emission showing some linear polarization (Dyks et al. 2004; Venter et al. 2012). In a study of $30 \gamma$-ray MSPs, Espinoza et al. (2013) indeed find that MSPs with aligned profiles have the largest inferred magnetic fields at their light cylinders. ${ }^{18}$ They furthermore find that those MSPs have the steepest radio spectra, with a probability of less than $1 \%$ of originating from the same spectral distribution as other ( $\gamma$-ray) MSPs.

In modeling the light curves of $\gamma$-ray MSPs with a variety of magnetospheric models, Johnson et al. (2014) find that the MSPs with radio and $\gamma$-ray peak alignment within 0.1 in rotational phase, are best fitted by outer gap and slot gap models (see also Grenier \& Harding 2015 and references therein). PSR J1552+5437 supports the hypothesis that to find

\footnotetext{
16 http://www.atnf.csiro.au/research/pulsar/psrcat

17 http://www.swift.psu.edu/unassociated

$18 B_{\mathrm{lc}} \propto P^{-5 / 2} \dot{P}^{1 / 2}$; about $5 \times 10^{4} \mathrm{G}$ for PSR J1552+5437 and typically $>10^{4}$ for $\gamma$-ray MSPs, with PSR B1937+21 having $\sim 10^{6} \mathrm{G}$.
} 
the fastest-spinning pulsars we need to find the MSPs with the steepest spectra; further surveys with LOFAR and other lowfrequency radio telescopes (as well as SKA-Low in the future) are instrumental in this quest.

In further support of this hypothesis, Frail et al. (2016) find that of the 16 pulsars with the steepest spectra (spectral index $<-2.5)$ in spectral measurements of 200 GMRT-detected pulsars (at $150 \mathrm{MHz}$; in the same frequency range as our LOFAR survey), 12 are MSPs, and all but one are $\gamma$-ray MSPs. A new detailed population study, similar to the ones performed by Kramer et al. (1998) or Bates et al. (2013), but including the findings of low-frequency surveys for MSPs, can establish whether the faster-spinning pulsars truly have steeper spectra on average, and whether the spectral distributions of slow pulsars and ( $\gamma$-ray) MSPs diverge.

We thank Rob Archibal, Maura Pilia, David Smith, Joris Verbiest, the Fermi LAT team, and the referee for useful discussions; LOFAR Science Operations and Support for their help in scheduling and effectuating these observations; and Andrew Lyne for obtaining observations with the Lovell Telescope. The research leading to these results has received funding from the European Research Council under the European Union's Seventh Framework Programme (FP7/ 2007-2013)/ERC grant agreement No. 337062 (DRAGNET; PI: Hessels). J.W.T.H. is an NWO Vidi fellow. LOFAR, the Low-Frequency Array designed and constructed by ASTRON, has facilities in several countries, that are owned by various parties (each with their own funding sources), and that are collectively operated by the International LOFAR Telescope (ILT) foundation under a joint scientific policy. Survey observations were taken under proposal LC5_002 (PI: Pleunis), timing observations under proposals DDT5_003 (PI: Pleunis) and LT5_003 (PI: Verbiest). Pulsar research at the Jodrell Bank Centre for Astrophysics and the observations using the Lovell Telescope are supported by a consolidated grant from the STFC in the UK. The Nançay Radio Observatory is operated by the Paris Observatory, associated with the French Centre National de la Recherche Scientifique (CNRS). The FermiLAT Collaboration acknowledges generous ongoing support from a number of agencies and institutes that have supported both the development and the operation of the LAT as well as scientific data analysis. These include the National Aeronautics and Space Administration and the Department of Energy in the United States, the Commissariat à l'Energie Atomique and the Centre National de la Recherche Scientifique/Institut National de Physique Nucléaire et de Physique des Particules in France, the Agenzia Spaziale Italiana and the Istituto Nazionale di Fisica Nucleare in Italy, the Ministry of Education, Culture, Sports, Science and Technology (MEXT), High Energy Accelerator Research Organization (KEK) and Japan Aerospace Exploration Agency (JAXA) in Japan, and the K. A. Wallenberg Foundation, the Swedish Research Council and the Swedish National Space Board in Sweden. Additional support for science analysis during the operations phase is gratefully acknowledged from the Istituto Nazionale di Astrofisica in Italy and the Centre National d'Etudes Spatiales in France. This work performed in part under DOE Contract DE-AC0276 SF00515.

Facilities: LOFAR, Fermi, GBT, Lovell, NRT.

Software: cdmt, PRESTO, PSRCHIVE, Astropy.

\section{ORCID iDs}

Z. Pleunis (ํ) https://orcid.org/0000-0002-4795-697X

J. W. T. Hessels (i) https://orcid.org/0000-0003-2317-1446

F. Camilo (1) https://orcid.org/0000-0002-1873-3718

\section{References}

Abdo, A. A., Ajello, M., Allafort, A., et al. 2013, ApJS, 208, 17 Acero, F., Ackermann, M., Ajello, M., et al. 2015, ApJS, 218, 23 Acero, F., Ackermann, M., Ajello, M., et al. 2016, ApJS, 223, 26 Arzoumanian, Z., Gendreau, K. C., Baker, C. L., et al. 2014, Proc. SPIE, 9144, 914420

Atwood, W. B., Abdo, A. A., Ackermann, M., et al. 2009, ApJ, 697, 1071

Bassa, C. G., Pleunis, Z., \& Hessels, J. W. T. 2017a, A\&C, 18, 40

Bassa, C. G., Pleunis, Z., Hessels, J. W. T., et al. 2017b, ApJL, 846, L20

Bates, S. D., Lorimer, D. R., \& Verbiest, J. P. W. 2013, MNRAS, 431, 1352 Bhat, N. D. R., Cordes, J. M., Camilo, F., Nice, D. J., \& Lorimer, D. R. 2004, ApJ, 605, 759

Camilo, F., Kerr, M., Ray, P. S., et al. 2015, ApJ, 810, 85

Clark, C. J., Wu, J., Pletsch, H. J., et al. 2017, ApJ, 834, 106

Cordes, J. M., \& Lazio, T. J. W. 2002, arXiv:astro-ph/0207156

Cromartie, H. T., Camilo, F., Kerr, M., et al. 2016, ApJ, 819, 34

de Jager, O. C., Raubenheimer, B. C., \& Swanepoel, J. W. H. 1989, A\&A, 221,180

Dyks, J., Harding, A. K., \& Rudak, B. 2004, ApJ, 606, 1125

Espinoza, C. M., Guillemot, L., Çelik, Ö., et al. 2013, MNRAS, 430, 571

Frail, D. A., Jagannathan, P., Mooley, K. P., \& Intema, H. T. 2016, ApJ, 829,119

Grenier, I. A., \& Harding, A. K. 2015, CRPhy, 16, 641

Hamaker, J. P. 2006, A\&A, 456, 395

Hessels, J. W. T., Ransom, S. M., Stairs, I. H., et al. 2006, Sci, 311, 1901

Hobbs, G. B., Edwards, R. T., \& Manchester, R. N. 2006, MNRAS, 369, 655

Hotan, A. W., van Straten, W., \& Manchester, R. N. 2004, PASA, 21, 302

Johnson, T. J., Venter, C., Harding, A. K., et al. 2014, ApJS, 213, 6

Keith, M. J., Coles, W., Shannon, R. M., et al. 2013, MNRAS, 429, 2161

Kerr, M. 2011, ApJ, 732, 38

Kerr, M., Ray, P. S., Johnston, S., Shannon, R. M., \& Camilo, F. 2015, ApJ, 814,128

Kondratiev, V. I., Verbiest, J. P. W., Hessels, J. W. T., et al. 2016, A\&A, 585, A128

Kramer, M., Xilouris, K. M., Lorimer, D. R., et al. 1998, ApJ, 501, 270

Lorimer, D. R., \& Kramer, M 2012, Handbook of Pulsar Astronomy (Cambridge: Cambridge Univ. Press)

Manchester, R. N., Hobbs, G. B., Teoh, A., \& Hobbs, M. 2005, AJ, 129, 1993

Nice, D. J., \& Taylor, J. H. 1995, ApJ, 441, 429

Noutsos, A., Sobey, C., Kondratiev, V. I., et al. 2015, A\&A, 576, A62

Pletsch, H. J., \& Clark, C. J. 2015, ApJ, 807, 18

Pletsch, H. J., Guillemot, L., Fehrmann, H., et al. 2012, Sci, 338, 1314

Ransom, S. M. 2001, PhD thesis, Harvard Univ.

Ray, P. S., Abdo, A. A., Parent, D., et al. 2012, arXiv:1205.3089

Ray, P. S., Kerr, M., Parent, D., et al. 2011, ApJS, 194, 17

Saz Parkinson, P. M., Xu, H., Yu, P. L. H., et al. 2016, ApJ, 820, 8

Shklovskii, I. S. 1970, SvA, 13, 562

Stappers, B. W., Hessels, J. W. T., Alexov, A., et al. 2011, A\&A, 530, A80

Stroh, M. C., \& Falcone, A. D. 2013, ApJS, 207, 28

van Haarlem, M. P., Wise, M. W., Gunst, A. W., et al. 2013, A\&A, 556, A2

Venter, C., Johnson, T. J., \& Harding, A. K. 2012, ApJ, 744, 34

Wang, J. B., Hobbs, G., Coles, W., et al. 2015, MNRAS, 446, 1657

Yao, J. M., Manchester, R. N., \& Wang, N. 2017, ApJ, 835, 29 